\title{
A propósito de un caso de psicosis temprana: un nuevo paradigma en salud mental.
}

About a case of early psychosis: a new paradigm in mental health.

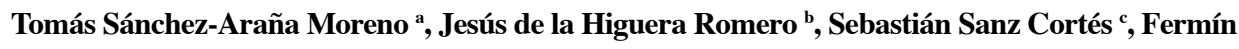

Pérez Pérez ${ }^{\mathrm{d}}$.

${ }^{a}$ FEA de Psiquiatría. Unidad de Rehabilitación. UGC de Salud Mental. Hospital Universitario de Puerto Real, España. ${ }^{b} F E A$ de Psicología clínica. Unidad de Rehabilitación. UGC de Salud Mental. Hospital Universitario de Puerto Real, España. ${ }^{c} F E A$ de Psiquiatría. Unidad de Rehabilitación. UGC de Salud Mental. Hospital Universitario de Puerto Real, España. ${ }^{d} F E A$ de Psicología clínica. Unidad de Rehabilitación. UGC de Salud Mental. Hospital Universitario de Puerto Real, España.

Correspondencia: Tomás Sánchez-Araña Moreno (tsam567@gmail.com)

Recibido: 15/07/2011; aceptado: 03/11/2011

RESUMEN: La intervención precoz en las fases tempranas de las psicosis ha adquirido una creciente relevancia en los últimos años debido a sus potenciales implicaciones sobre la clínica y la evolución de la enfermedad. Es conocido que tras un primer episodio psicótico hay un "periodo crítico" que se extiende a los cinco años siguientes, siendo esta una fase de alta vulnerabilidad. El abordaje integral e intensivo durante este periodo tiene como finalidad optimizar el proceso de recuperación ayudando al paciente y a su familia a reconstruir sus vidas y evitar recaídas. A continuación exponemos la gestión de un caso en un reciente programa de "intervención precoz en psicosis" implementado en nuestra unidad de rehabilitación.

PALABRAS CLAVE: Trastornos Psicóticos, Terapia Combinada, Salud Mental.
ABSTRACT: Early intervention in the early phases of psychosis has become increasingly relevant in recent years because of their potential clinical implications for the evolution of the disease. It is known that after a first psychotic episode is a "critical period" that extends to five years, this being a period of high vulnerability. Comprehensive and intensive approach during this period aims at optimizing the recovery process by helping the patient and his family rebuild their lives and prevent relapse. Below are the management of a case in a recent program of "early intervention in psychosis" implemented in our rehabilitation unit.

KEY WORDS: Psychotic Disorders, Combined Modality Therapy, Mental Health.

Antecedentes, presentación y abordaje del caso.

Paciente de 22 años de edad, soltero y sin hijos. Es el $3^{\circ}$ de una fratría de 4 hermanos. Estudiante de tercer año de una licenciatura universitaria. Convive en el domicilio familiar con sus padres y su hermano menor. Sin antecedes psiquiátricos personales ni familiares. Tampoco hay que reseñar patología somática alguna pero sí cabe destacar consumo de cannabinoides ocasional los fines de semana.

Ingresa en la Unidad de Agudos de nuestro hospital de referencia a finales de 2008 por presentar trastorno psicótico agudo de tipo esquizofreniforme (CIE-10, F23.2) donde permanece hospitalizado durante 15 días. A su alta, con prescripción antipsicótica de paliperidona $6 \mathrm{mgr} /$ día, es derivado a la Unidad de Rehabilitación 
de nuestra área donde se incluye al paciente en un programa de "intervención precoz en psicosis".

Óptimo ajuste psicosocial premórbido y una dinámica familiar "sana" con ausencia de alta emoción expresada. Estimamos una DUI (Duration of Untreated Illness) y una DUP (Duration of Untreated Psicosis) "muy cortas", que de entrada suponían factores de buen pronóstico. La DUI o periodo transcurrido entre la fase prodrómica y el inicio del tratamiento fue de aproximadamente un año. En este periodo destacó la instauración insidiosa de cambios en la percepción de si mismo y del mundo en general, dificultades cognitivas en la atención y memoria, bajo rendimiento académico así como tendencia al aislamiento. La DUP o periodo transcurrido entre la fase con síntomas positivos y el inicio del tratamiento se estimó en 3 meses.

\section{Evaluación:}

El proceso de evaluación se organizo para obtener información sobre 6 áreas de relevancia para la estructuración del plan individualizado de tratamiento (1). Las áreas en cuestión fueron las siguientes:

1.- Perfil psicopatológico: se utilizaron 2 instrumentos de evaluación, el BPRS-24 y el PSYRATS, este último para delimitar de manera más precisa los parámetros concretos a nivel de sintomatología.

2.- Perfil cognitivo: se evaluaron funciones cognitivas básicas como la atención, la memoria verbal, la resistencia a la interferencia cognitiva o el funcionamiento ejecutivo. Los instrumentos específicos de evaluación fueron:

-El test de Aprendizaje Verbal España-Complutense (TAVEC)

- Continuous Performance Tests

-Wisconsin Card Shorting Test (WCST)

-Test de Interferencia Neurocognitiva de Stroop

-Trail Making (formas A y B)

3.-Perfil funcional: Se utilizó la versión castellana de la Social Functioning Scale (SFS).

4.- Autoestima: para evaluar aspectos vinculados a la recuperación de la percepción de control y de la capacidad personal de afrontamiento se utilizó la Escala de Autoestima de Rosemberg.

5.- Calidad de vida percibida: utilizamos la EuroQol 5 D

6.- Capacidad de afrontamiento familiar: utilizamos el Family Coping Questionnaire. 
NOTAS CLÍNICAS

La evaluación se realizó en dos cortes evaluativos, uno basal inicial y otro post-intervención.

\section{Tratamiento:}

El plan individualizado de tratamiento se estructuró sobre 3 ejes de actuación:

1.-Psicoterapia individual de orientación cognitivo conductual orientada hacia la recuperación.

En nuestro caso seguimos la filosofía y orientaciones concretas explicitadas en el programa COPE (Cognitive Psychotherapy for Early Psychosis) (2) que es un procedimiento con evidencia empírica de respaldo y que incluye en su formulación una filosofía de base orientada hacia la optimización de los recursos personales y el empoderamiento. Estos aspectos nos parecen de vital importancia en el trabajo psicoterapéutico con este tipo particular de población.

2.- Abordaje psicoeducativo familiar.

Utilizamos la Terapia Familiar Comportamental de Falloon (3). Este procedimiento nos parece especialmente interesante porque permite trabajar con todos los miembros de la unidad familiar y no solo mejorar su conocimiento sobre la enfermedad sino también actuar de manera específica sobre las habilidades individuales de comunicación y solución de problemas, estos últimos muy vinculados a los niveles de emoción expresada.

3.- Tratamiento psicofarmacológico.

Mantuvimos tratamiento psicofarmacológico con paliperidona $6 \mathrm{mg} / \mathrm{día} \mathrm{du}-$ rante 12 meses para, tras una remisión sintomática completa, ir disminuyendo la dosis hasta la supresión completa a los 18 meses.

Es recomendable la utilización de medicación antipsicótica atípica, a la menor dosis posible, por su mejor tolerancia y menor riesgo de síntomas extrapiramidales que permitan garantizar una buena adherencia.

En líneas generales podemos hacer notar que el efecto de la intervención programada sobre la persona afectada fue positivo, se produjo una mejora en la conciencia de enfermedad y en sus habilidades de afrontamiento ante situaciones estresantes. La adherencia al tratamiento y la abstinencia en el consumo de cannabis fueron determinantes en la recuperación completa del cuadro. Hoy en día nuestro paciente está a punto de licenciarse y lleva una vida completamente normalizada en todos los ámbitos. 


\section{Discusión.}

La intervención descrita se contextualiza dentro del Programa de Intervención en Primeros Episodios (PIPE) que actualmente estamos desarrollando dentro de nuestra Unidad de Gestión Clínica de Salud Mental, adscrita al Hospital Universitario de Puerto Real (4). Más allá de describir las peculiaridades del programa si nos gustaría hacer mención a la necesidad y relevancia de este tipo de intervenciones precoces de cara a mejorar el pronóstico de estos cuadros. Hoy día parece suficientemente claro la necesidad de articular respuestas a través de servicios que provean de una oferta terapéutica, comprensiva, accesible y que se oriente hacia la recuperación y el empoderamiento personal. No existen dudas sobre el hecho de que la intervención precoz puede contribuir a evitar el deterioro biológico, social y psicológico que por lo general puede producirse en los años siguientes al comienzo del trastorno psicótico, siendo los beneficios potenciales de este tipo de intervención: la disminución de la morbilidad, una recuperación más rápida, mejor pronóstico, conservación de las habilidades psicosociales, conservación del apoyo familiar y social, así como la menor necesidad de hospitalización (5). Humildemente hemos pretendido trasladar una visión algo más optimista que la de los grandes maestros Kraepelin (6) y Minkowski (7), quienes con el saber y la elegancia que les caracterizaban abogaban por la evolución inexorable de la esquizofrenia a un estado deficitario.

\section{BIBLIOGRAFÍA:}

(1) Stone, W.S., Faraone, S.V. y Tsuang, M.T. Intervención clínica precoz y prevención de la esquizofrenia. Barcelona: J\&C Ediciones Médicas, 2004.

(2) Cognitive-Oriented Psychotherapy for Early Psychosis. Early Psychosis Prevention and Intervention Centre. EPPIC. Manual 4 in a series of early psychosis manual). 2002.

(3) Falloon, IRH; Laporta, M; Fadden, G \& Graham-Hole, V. Managing Stress in Families: Cognitive and Behavioural Strategies for Enhancing Coping Skilss.Routledge.1993.

(4) Edwards, J., McGorry, P. D. La intervención precoz en la psicosis: Guía para la creación de servicios de intervención precoz en la psicosis. Madrid: Fundación para la investigación y el tratamiento de la esquizofrenia y otras psicosis, 2004.

(5) AEN. Consenso sobre atención temprana a la psicosis de la Asociación Española de Neuropsiquiatría. Madrid: Cuadernos Técnicos AEN , 2009.

(6) Kraepelin E. Dementia praecox and paraphrenia. Edinburgh, E \& S Livingstone; 1919.

(7) MINKOWSKI, Eugéne. La esquizofrenia, psicopatología de los enfermos y los esquizofrénicos. Fondo de Cultura Económica, Biblioteca de psicología, psiquiatría y psicoanálisis. México, 2000. 